\title{
Perturbation theory for the two-impurity Kondo problem- RKKY-induced vanishing of the Kondo temperature
}

\author{
W Loinaz†, J W Rasulf and P Schlottmann \\ $\uparrow$ Physics Department, University of Michigan, Ann Arbor, MI 48109, USA \\ † Department of Physics and Center for Materials Research and Technology, Florida State \\ University, Tallahassee, FL 32306, USA
}

Received 16 Noyember 1992 , in final form 24 February 1993

\begin{abstract}
We consider the two-impurity Kondo problem, in a basis in which the electron states are written in terms of their parity with respect to the midpoint between impurities. We consider the scattering of electrons by the spin-one complexes formed by the impurities, making use of the fact that the RKKY interaction is diagonal in this basis and acts in much the same way that a crystal field acts in the degenerate Kondo problem. By expanding the equations of motion to leading logarithmic order we show that the resulting Kondo temperature is reduced by an increasing antiferromagnetic RKKY interaction, and for one of the electron parity channels crosses the antiferromagnetic RKKY singlet. This happens when the RKKY interaction is of the order of the bare (no RKKY) Kondo temperature. We interpret this crossing as reflecting the divergence of Fermi liquid properties found in numerical studies along the lines of a phenomenological model introduced by two of the authors.
\end{abstract}

\section{Introduction}

Despite much progress in the last thirty years the problem of the competition between localized magnetic order and itinerant behaviour continues to confound theoretical and experimental investigators. Although the properties of single-impurity systems are by now understood, the simplest extension, namely the problem with two magnetic impurities that scatter surrounding conduction electrons, has generally been beyond the reach of the kind of techniques that resolved the single-impurity problem. Recent theoretical progress has, however, revealed a rich behaviour in the region where the tension between localized and itinerant behaviour is strongest, namely where the antiferromagnetic RKKY and Kondo energy scales are of the same order of magnitude.

As according to recent numerical renormalization group (RG) studies [1] and conformal invariance approaches [2] there exists an unstable fixed point separating the stable Kondo and antiferromagnetic fixed points. In the neighbourhood of this fixed point the Fermi liquid parameters that characterize the low-temperature behaviour show a divergence as the RKKY interaction $(I)$ approaches roughly twice the Kondo temperature $\left(T_{\mathrm{K}}\right)$. The divergence (as a function of $I-2 T_{K}$ ) shows up in the linear specific heat coefficient and the staggered susceptibility but not in the uniform susceptibility [1]. Furthermore, the expectation value $\left\langle S_{1} S_{2}\right\rangle$ is approximately $-1 / 4$ at this fixed point. While these results are of interest in their own right, the problem arises as to whether this behaviour can be understood from any more conventional viewpoints. This issue is particularly important for lattice problems and concentrated alloys as well as for spin glass systems in which the itinerant electron effects play a role. 
After the original numerical RG study, a simple model was proposed by two of the authors [3] that reproduced a number of features of the exact study. The model consisted of four spins, two of which represented the impurities, and two of which represented the on-site conduction electron spin densities. The on-site coupling between the electron and impurity was taken to be the single-ion Kondo temperature, so that the appropriate singlet-triplet splitting was obtained in the spirit of the phenomenology of Nozieres [4]. By including intersite couplings, this model was found to display a crossover between antiferromagnetic singlet and Kondo ground states as the couplings were varied. The crossover occurred at a value of the RKKY interaction equal to twice the Kondo temperature. At this point it was also found that the Kondo and antiferromagnetic singlets were degenerate with a third state, namely an odd-parity triplet.

As a consequence of the above degeneracies, operators that mix the odd-parity triplet to the singlets, such as the staggered susceptibility, would naturally diverge at such a point. On the other hand, mixing to the higher-lying even-parity triplet would not cause a divergence. Nevertheless, as a result of the degeneracy of the two singlets the specific heat would diverge. The fact that this simpie model recovers the broad qualitative features of the exact solution has spurred attempts to place this phenomenological model within a more rigorous manybody framework. Starting from the Anderson model, Saso [5] observed that by transforming the electron states into ones with even and odd parity with respect to the impurity centre of gravity, the model had formal similarities with the $\mathrm{f}^{1}-\mathrm{f}^{2}$ Anderson model. Applying a variational calculation to this problem, in which the Kondo and antiferromagnetic singlets could be explicitly examined, he investigated the crossover between the two ground states.

A similar calculation was performed by Yanasigawa [6], albeit for different coupling schemes, which allowed him to compare with numerical diagonalizations on small systems. It emerged from these variational and numerical calculations that the divergences in the response functions for the Kondo model were smeared out. The same behaviour was found by Sakai et al [7] in a numerical RG study of the two-impurity Anderson model. These authors tried a number of coupling schemes, in order to understand the discrepancies between the Kondo RG studies and Monte Carlo studies [8] of the two-impurity problem. They found a sensitive dependence on the coupling scheme, with the least amount of smearing for the Jones et al [1] choice of coupling (which in their more recent studies [7] has indeed turned into a divergence). They also found an asymmetry in the spectral densities of the even- and odd-parity channels if anything other than the parity- and energy-independent hybridization was used.

The qualitative differences between Kondo and Anderson model behaviour can be attributed in part at least to the existence of potential scattering in the latter but not in the former. Jones and Varma [9] carried out a numerical RG study incorporating potential scattering and indeed found a broadening of the divergence at $I=2 T_{\mathrm{K}}$. Within a slaveboson mean-field theory this broadening was related to scattering phase shifts, which take quantized values at the fixed points when there is particle-hole symmetry $[10,11]$.

In this paper we attempt to study the problem without potential scattering from the conventional perturbation viewpoint. We will not try to obtain the exact ground state of the two-impurity problem; rather we seek to understand the effects of the Kondo spin dynamics, by examining the energy scale characterizing the breakdown of high-energy perturbation theory and the transition to the Fermi liquid regime. The validity of our results is therefore restricted to the perturbative regime.

As in the above-mentioned variational studies we shall work within the even- and oddparity state scheme in which the RKKY interaction is diagonal and acts as a crystal field splitting does in the normal single-impurity Kondo problem. By separating the impurity 
states into the singlet and triplet states and rewriting the Kondo scattering terms in terms of scatterings of conduction electron states from these impurity states, we can apply the normal techniques of second-order perturbation theory in the Kondo coupling strength. We shall adopt the equation of motion approach since for the singlet and triplet representation it is the most straightforward. Applying this method in the vertices for scattering of evenand odd-parity electron states within and between the triplet and singlet impurity states we shall obtain the effective Kondo temperature at which perturbation theory breaks down.

A schematic description of the problem is indicated in figure 1. For zero RKKY splitting we expect the Kondo temperature to be that of the isolated impurities. As the splitting is switched on the Kondo temperature changes, reflecting the influence of magnetic interactions on the Kondo effect. The singlet state is pulled out from undemeath the triplet and under the right conditions one might expect to see the level crossing (of the type mentioned above) result if the splitting is large enough. A closing of the gap between the antiferromagnetic singlet and Kondo binding energy might then reflect an increase of the Fermi liquid parameters characterizing the low-temperature behaviour.
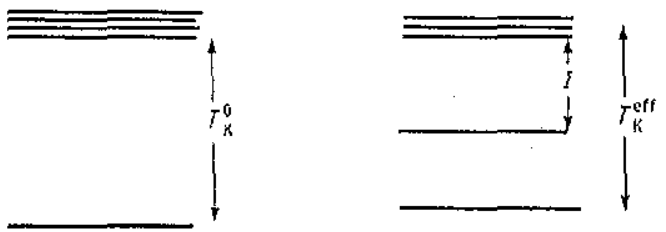

Figure 1. Schematic illustration of the position of the Kondo singlet relative to the $S=1$ complex formed by the two impurities before and after the introduction of an RKKY splitting.

In the following section we formulate the problem in terms of even- and odd-parity electron states and derive the equations of motion for the vertices. After some elimination we are able to reduce these equations of motion to a form suitable for numerical treatment. We shall trace the behaviour of the effective Kondo temperature, starting from its known zero RKKY value, as the RKKY interaction is increased.

\section{Formulation}

Our starting point is the conventional two impurity Kondo model

$$
H=\sum_{k \sigma} \epsilon_{k} c_{k \sigma}^{+} c_{k \sigma}+J \sum_{k, k_{1}, \sigma, \sigma_{t}}\left[\mathrm{e}^{\mathrm{i}\left(k-k_{1}\right) R_{1}} S_{1}+\mathrm{e}^{\mathrm{i}\left(k-k_{1}\right) R_{2}} S_{2}\right] c_{k \sigma}^{+} \sigma c_{k_{1} \sigma_{\mathrm{t}}}
$$

where $c_{k r}$ labels the electron states with wavevector $k$ and spin $\sigma, S_{1}$ and $S_{2}$ label the two impurity spins at positions $R_{1}$ and $R_{2}$ respectively. By integrating out the angles around the line joining the two impurities we can rewrite the electron states as a 1D problem, namely in terms of forward $\left(a_{q \sigma}\right)$ and backward $\left(b_{q \sigma}\right)$ states. Relabelling the backward states so that they have the same energy as the forward states, replacing the wavevectors in the exponentials by their values at the Fermi surface, and defining the even-and odd-parity electron states by the relation

$$
c_{k, p, \sigma}=\left(a_{k \sigma}+p b_{k \sigma}^{+}\right) / \sqrt{2}
$$


we can write the resulting two-impurity Hamiltonian in the form

$$
\begin{array}{r}
H=\sum_{k, p, \sigma} \epsilon_{k} c_{k, p, \sigma}^{+} c_{k, p, \sigma}+J\left(S_{1}+S_{2}\right) \sum_{k, k_{1}} c_{k, p, \sigma}^{+} \sigma c_{k_{1}, p, \sigma}\left(1+p \cos \left(k_{\mathrm{F}} R\right)\right) \\
+\mathrm{i} \sin \left(k_{\mathrm{F}} R\right) J\left(S_{1}-S_{2}\right) \sum_{k_{1, k_{1}, p}}\left[c_{k,-, \sigma}^{+} c_{k_{3},+, \sigma}-c_{k_{,}, \sigma}^{+} c_{k_{1},-, \sigma}\right]
\end{array}
$$

where $R=\left|R_{1}-R_{2}\right|$ denotes the impurity separation. It remains to transform the impurity spin operators into a basis where the RKKY is diagonal. To this end we define the singlet and triplet states as follows:

$$
\begin{array}{ll}
\left.\left.s^{+}=[1+-)-1-+\right\rangle\right] / \sqrt{2} & \left.t_{-}^{+}=1--\right\} \\
\left.t_{0}^{+}=[1+-)+|-+\rangle\right] / \sqrt{2} & \left.t_{+}^{+}=1++\right\rangle .
\end{array}
$$

In terms of the above states the interaction part of the Hamiltonian reads

$$
\begin{aligned}
& H_{\mathrm{I}}=(J / 2)\left(t_{+}^{+} t_{+}-t_{-}^{+} t_{-}\right) \sum_{k_{,} k_{1}, p, \sigma} \sigma c_{k, p, \sigma}^{+} c_{k_{1}, p, \sigma}\left(1+p \cos \left(k_{\mathrm{F}} R\right)\right) \\
& +\mathrm{i} \sin \left(k_{F} R\right)(J / \sqrt{2})\left(s^{+} t_{0}+t_{0}^{+} s\right) \sum_{k, k_{1}, \sigma} \sigma\left(c_{k_{,},-\sigma}^{+} c_{k_{1},+, \sigma}-c_{k_{,}, \sigma}^{+} c_{k_{1},-, \sigma}\right) \\
& +(J / 2)\left(t_{0}^{+} t_{-}+t_{+}^{+} t_{0}\right) \sum_{k, k_{1}, p} c_{k, p,-}^{+} c_{k_{1}, p_{.}-}\left(1+p \cos \left(k_{\mathrm{F}} R\right)\right) \\
& +\mathrm{i} \sin \left(k_{\mathrm{F}} R\right)(J / \sqrt{2})\left(s^{+} t_{-}+t_{+}^{+} s\right) \sum_{k_{1, k_{1}}}\left(c_{k_{,},-,}^{+} c_{k_{1},+,+}-c_{k_{,} \Psi_{-}-}^{+} c_{k_{1},-+}\right) \\
& +(J / 2)\left(t_{0}^{+} t_{+}+t_{-}^{+} t_{0}\right) \sum_{k_{,} k_{1}, p} c_{k, p_{1}+}^{+} c_{k_{1}, p_{1}-}\left(1+p \cos \left(k_{\mathrm{F}} R\right)\right) \\
& +\mathrm{i} \sin \left(k_{\mathrm{F}} R\right)(J / \sqrt{2})\left(t_{-}^{+} s+s^{+} t_{+}\right) \sum_{k, k_{1}}\left(c_{k_{1},+,}^{+} c_{k_{1},+,-}-c_{k,+,+}^{+} c_{k_{1},-,-}\right) .
\end{aligned}
$$

It should be noted that it is the order of the singlet and triplet operators that is relevant-they are projection operators and the notation is not supposed to imply any particular commutation relations. In terms of these operators the RKKY interaction becomes

$$
H_{\mathrm{RKKY}}=E_{\mathrm{S}} s^{+} s+E_{\mathrm{T}}\left[t_{+}^{+} t_{+}+t_{0}^{+} t_{0}+t_{-}^{+} t_{-}\right]
$$

where $E_{\mathrm{S}}-E_{\mathrm{T}}=I$, the unrenormalized RKKY interaction. It remains to define the vertices for scattering of the conduction electrons from these impurity states. They are as follows.

$$
\begin{aligned}
& \Gamma_{t_{0, s}}=\sum_{k}\left\langle c_{k,-.+} t_{0}^{+} s ; c_{k_{1},+,+}^{+}\right\rangle_{\mathrm{i} \omega_{n}} \quad \Gamma_{t_{-} t_{0}}=\sum_{k}\left\langle c_{k_{1}+,-} \sqrt{2} t_{-}^{+} t_{0} ; c_{k_{1},+,+}^{+}\right\rangle_{\mathrm{i} \omega_{n}} \\
& \Gamma_{s, t_{n}}=\sum_{k}\left(c_{k,-,+} s^{+} t_{0} ; c_{k_{1},+,+}^{+}\right)_{i \omega_{n}} \quad \Gamma_{t_{+} t_{+}}=\sum_{k}\left\langle c_{k_{1}+,+} t_{+}^{+} t_{+} ; c_{k_{1},+,+}^{+}\right\rangle_{\mathrm{i} \omega_{n}} \\
& \left.\Gamma_{t_{s} x}=\sum_{k}\left\langle c_{k_{s}-} \sqrt{2} t_{-}^{+} s ; c_{k_{1},+,+}^{+}\right\rangle_{\mathrm{i} \omega_{n}} \quad \Gamma_{s, t_{+}}=\sum_{k} c_{k_{,},-,-}\left(-\sqrt{2} s^{+} t_{+}\right) ; c_{k_{1},+,+}^{+}\right)_{\mathrm{i} \omega_{n}} \\
& \Gamma_{t_{0, t_{+}}}=\sum_{k}\left\{c_{k,+,-} \sqrt{2} t_{0}^{+} t_{+} ; c_{k_{1},+,+}^{+}\right\}_{\mathrm{i} \omega_{n}} \quad \Gamma_{s, s}=\sum_{k}\left\langle c_{k,+,+} s^{+} s ; c_{k_{1},+,+}^{+}\right\rangle_{\mathrm{i} \omega_{n}} \\
& \Gamma_{t_{-} t_{-}}=\sum_{k}\left\langle c_{k_{1}+,+}\left(-t_{-}^{+} t_{-}\right) ; c_{k_{1}+t_{1}+}^{+}\right\rangle_{i \omega_{n}} \quad \Gamma_{t_{0} t_{0}}=\sum_{k}\left\langle c_{k,+,+} t_{0}^{+} t_{0} ; c_{k_{1},+,+}^{+}\right\rangle_{\mathrm{i} \omega_{n}} .
\end{aligned}
$$


We have chosen electron states of even parity as the final states-we will return to the odd-parity states later. For the moment all that matters is that these are the vertices that arise when we calculate the equations of motion of the even-parity single-particle Green function i.e.

$$
\begin{gathered}
\left\langle\left[c_{k,+,+}, H\right] ; c_{k_{t}, \tau_{+}+}^{+}\right\rangle=\sum_{k_{2}}\left[\left(J_{+}^{-} / 2 N\right)\left(\Gamma_{t_{+} t_{+}}+\Gamma_{t_{-} t_{-}}+\Gamma_{t_{0} t_{+}}+\Gamma_{t_{-} t_{0}}\right)\right. \\
\left.+\mathrm{i}(J / 2 N)\left(\Gamma_{s, t_{0}}+\Gamma_{t_{0} s}+\Gamma_{t_{-} s}+\Gamma_{s t_{+}}\right)\right] .
\end{gathered}
$$

We then proceed to evaluate the equations of motion for the above Green functions in the standard manner, decoupling the expressions resulting on the right-hand side in complete analogy to the Nagaoka approximation [12] used originally for the single-site Kondo problem. We illustrate the procedure on the vertex $\Gamma_{10, s}$

$$
\begin{aligned}
\left\langle\left[c_{k_{-},+}+t_{0} s, H_{1}\right] ;\right. & \left.c_{k_{1},+,+}^{+}\right\rangle=-\left(J_{+}^{-} / 2 n_{k}\right) \sum_{k_{2}}\left\langle c_{k_{2},-,-} t_{-}^{+} s ; c_{k_{1},+,+}^{+}\right\rangle \\
& -\mathrm{i}\left(J_{-} / 2 n_{k}\right) \sum_{k_{2}}\left\langle c_{k_{1},+,-} t_{0}^{+} t_{-} ; c_{k_{1},+,+}^{+}\right\rangle \\
& +\mathrm{i}\left(J_{-} / 2 n_{k}\right) \sum_{k_{2}}\left[\left\langle c_{k_{2},+,+} t_{0}^{+} t_{0} ; c_{k_{1},+,+}^{+}\right\rangle-\left\langle c_{k_{2},+,+} s^{+} s ; c_{k_{1},+,}^{+}\right\rangle\right]
\end{aligned}
$$

where we have replaced products of electron operators by average occupation numbers as, for example,

$$
c_{k_{1},+, \sigma}^{+} c_{k_{a},-, \sigma} c_{k,+,-} \rightarrow-\delta_{k, k_{1}}\left[1-n_{k,+,-}\right] c_{k_{a},-, \sigma} \delta_{\sigma_{0}-}
$$

and terms such as

$$
c_{k,+,-} c_{k_{1},+, \sigma}^{+} c_{k_{a},-, \sigma} \rightarrow n_{k_{1}} c_{k_{a},-,-} \delta_{k_{1}} \delta_{\sigma-}
$$

We have introduced the shorthand

$$
J_{+}^{+}=J\left(1+\cos k_{\mathrm{F}} R\right) \quad J_{-}=J \sin \left(k_{\mathrm{F}} R\right)
$$

and the occupation number $n_{k}=\left\langle c_{k, p, \sigma}^{+} c_{k, p, \sigma}\right\}=1-f(k)$ where $f(k)$ denotes the Fermi function. In addition to the above terms other terms are produced on the RHS, but these involve combinations of the one-electron Green function and frequency integrations over the vertices in (7). As such, they provide non-logarithmic corrections that would only matter if we were to attempt a full solution of the problem. Since we know from experience with the Kondo problem that the Nagaoka approximation is only adequate as far as the leading logarithmic divergences, we ignore these terms. Only the terms listed above relate to logarithmic coefficients of the vertices listed in equation (7), and in order to obtain the Kondo energy scale for our problem only these terms are necessary. 


\section{Results}

The full set of equations obtained in this way is given by

$$
\begin{aligned}
& \Gamma_{t_{0} s}=-\left(J_{+}^{-} M / 2\right) \Gamma_{t_{-} s}-\left(\mathrm{i} J_{-} M / 2\right) \Gamma_{t_{0} t_{+}}+\left(\mathrm{i} J_{-} M / 2\right)\left(\Gamma_{t_{0} t_{0}}-\Gamma_{s s}\right) \\
& \Gamma_{t_{-} t_{-}}=-\left(J_{+}^{+} L / 2\right) \Gamma_{t_{-} t_{0}}+\mathrm{i} J_{-} L \Gamma_{t_{-} s} / 2 \\
& \Gamma_{t_{0} t_{+}}=-J_{+}^{+} L \Gamma_{t_{0} t_{+}} / 2-J_{+}^{+} L \Gamma_{t_{+} t_{+}}+J_{+}^{+} L \Gamma_{t_{0} t_{0}}+\mathrm{i} J_{-} L \Gamma_{t_{0} s}+\mathrm{i} J_{-} L \Gamma_{s t_{+}} / 2 \\
& \Gamma_{t_{-} t_{0}}=-\left(J_{+}^{-} L / 2\right) \Gamma_{t_{-} t_{0}}+\left(\mathrm{i} J_{-} L / 2\right) \Gamma_{t_{-} s}-J_{+}^{+} L\left(\Gamma_{t_{-} t_{-}}+\Gamma_{t_{0} t_{0}}\right)+\mathrm{i} J_{-} L \Gamma_{s t_{0}} \\
& \Gamma_{s t_{+}}=-\left(J_{+}^{-} N / 2\right) \Gamma_{s t_{+}}-\left(\mathrm{i} J_{-} N / 2\right) \Gamma_{t_{0} t_{+}}-J_{+}^{-} N \Gamma_{s t_{0}}-\mathrm{i} J_{-} N \Gamma_{t_{+} t_{+}}+\mathrm{i} J_{-} N \Gamma_{s s} \\
& \Gamma_{s t_{0}}=-\left(J_{+}^{-} N / 2\right) \Gamma_{s t_{+}}-\left(\mathrm{i} J_{-} N / 2\right) \Gamma_{t-t_{0}}+\left(\mathrm{i} J_{-} N / 2\right)\left(\Gamma_{s s}-\Gamma_{t_{0} t_{0}}\right) \\
& \Gamma_{t_{+} t_{+}}=-\left(J_{+}^{+} / 2\right) L \Gamma_{t_{0} t_{+}}+\left(\mathrm{i} J_{-} L / 2\right) \Gamma_{s, t_{+}} \\
& \Gamma_{t_{-} s}=-\left(J_{+}^{-} M / 2\right) \Gamma_{t_{-} s}-\mathrm{i} J_{-} \Gamma_{t_{-} t_{0}} M / 2-J_{+}^{-} M \Gamma_{t t_{0}}-\mathrm{i} J_{-} \Gamma_{t_{-t}} M-\mathrm{i} J_{-} \Gamma_{s s} M \\
& \Gamma_{s s}=-\left(\mathrm{i} J_{-} L / 2\right)\left(\Gamma_{s t_{t}}-\Gamma_{t_{0} s}\right)-\left(\mathrm{i} J_{-} L / 2\right) \Gamma_{s t_{+}}+\left(\mathrm{i} J_{-} L / 2\right) \Gamma_{t_{-} s} \\
& \Gamma_{t_{0} t_{0}}=-\left(\mathrm{i} J_{-} L / 2\right)\left(\Gamma_{t s_{0}}-\Gamma_{s t_{0}}\right)+\left(J_{+}^{+} L / 2\right)\left(\Gamma_{t_{0} t_{+}}-\Gamma_{t_{-} t_{0}}\right)
\end{aligned}
$$

where as before, we neglect terms on the RHS that do not contain products of vertices and logarithmic factors. Here the logarithmic factors are represented by

$$
\begin{aligned}
& L\left(\mathrm{i} \omega_{n}\right)=\sum_{k} n_{k} /\left(\mathrm{i} \omega_{n}-\epsilon_{k}\right)=\ln \left(-\mathrm{i} \omega_{n} / D\right) \\
& M\left(\mathrm{i} \omega_{n}\right)=\sum_{k} n_{k} /\left(\mathrm{i} \omega_{n}-\epsilon_{k}+E_{\mathrm{T}}-E_{\mathrm{S}}\right)=\ln \left[\left(-\mathrm{i} \omega_{n}+E_{\mathrm{T}}-E_{\mathrm{S}}\right) / D\right] \\
& N\left(\mathrm{i} \omega_{n}\right)=\sum_{k} n_{k} /\left(\mathrm{i} \omega_{n}-\epsilon_{k}+E_{\mathrm{S}}-E_{\mathrm{T}}\right)=\ln \left[\left(-\mathrm{i} \omega_{n}+E_{\mathrm{S}}-E_{\mathrm{T}}\right) / D\right] .
\end{aligned}
$$

For zero RKKY interaction, we can form the linear combinations

$$
\begin{aligned}
& \Gamma_{1}=\Gamma_{t_{0} s}+\Gamma_{s t_{0}}+\Gamma_{s t_{+}}+\Gamma_{t_{-s} s} \\
& \Gamma_{2}=\Gamma_{r_{0} t_{+}}+\Gamma_{t_{+} t_{+}}+\Gamma_{t_{-t}}+\Gamma_{t_{-t} t_{0}}
\end{aligned}
$$

which satisfy the much simpler equations

$$
\begin{aligned}
& \Gamma_{1}=-J_{+}^{-} N \Gamma_{1}-\mathrm{i} J_{-} N \Gamma_{2} \\
& \Gamma_{2}=-J_{+}^{+} N \Gamma_{2}+\mathrm{i} J_{-} N \Gamma_{1}
\end{aligned}
$$

which at zero temperature have the solution $\omega=D \exp \left(-\frac{1}{2} \rho J\right)=T_{\mathrm{K}}^{0}$, namely the expected Kondo temperature for a system of two non-interacting Kondo impurities. Such a set of equations could also have been obtained starting from the original representation of the model and examining the quantities

$$
\begin{aligned}
& \Gamma_{1}=\left\langle\left(c_{k,+,+}\left(S_{1}^{z}+S_{2}^{z}\right)+c_{k_{1},+,-}\left(S_{1}^{-}+S_{2}^{-}\right)\right) ; c_{k,+,+}^{+}\right\rangle_{z} \\
& \Gamma_{2}=\left\langle\left(c_{k_{,-.}+}\left(S_{1}^{z}-S_{2}^{z}\right)+c_{k,-,-,}\left(S_{1}^{-}-S_{2}^{-}\right) ; c_{k_{1,+,+}^{+}}^{+}\right\rangle_{z}\right.
\end{aligned}
$$


which, not surprisingly, is what the linear combinations (19) and (20) reduce to in the limit of zero splitting. This supports our need to support the RKKY interaction as a kind of crystal field splitting since non-Kondo RKKY terms are generated at higher order than is consistent with the present level of decoupling.

As regards the odd-parity electron states it is straightforward to derive a set of equations corresponding to (13), for vertices that are related to the odd-parity single-electron Green function. As is clear from the Hamiltonian, these equations yield a matrix identical to that in (13), except for the replacements $J_{+}^{+} \leftrightarrow J_{+}^{-}$and $J_{-} \leftrightarrow-J_{-}$. Hermiticity ensures that the latter replacement is of no consequence.

Retuming to the full set of equations (13) we find that they can readily be reduced to an involved pair of equations relating to the vertices $\Gamma_{t_{0} t_{+}}$and $\Gamma_{t_{-} t_{0}}$, which has to be treated numerically. It is still straightforward to follow the evolution of the Kondo pole $\omega=T_{\mathrm{K}}^{0}$ as the splitting is varied. As a check, we obtain the same results using the algebraic manipulation program REDUCE directly on the set of equations in (13). In fact we find that the evolution of the Kondo pole is obtained from the vanishing of a single factor of the determinant of (13). For the even-parity states the effective Kondo temperature is given for general RKKY splitting by the solution of

$$
\begin{aligned}
1+J_{+}^{+} L+J_{+}^{-}(M+N)-J_{+}^{+} J_{+}^{-} L(M+N)+2 J_{+}^{-2} M N \\
-2 J_{+}^{-} J_{+}^{+2} L^{2}(M+N)-4 J_{+}^{+} J_{+}^{-2} L M N=0
\end{aligned}
$$

while for the odd-parity states the above-mentioned interchanging of $J_{+}^{-}$and $J_{+}^{+}$has to be made.

Turning to the solution of (23) we note that the determinant is invariant under a change of sign of the RKKY splitting. This would appear to imply that the physics is the same whether the RKKY interaction is ferromagnetic or antiferromagnetic. In fact for the former case it is known that the present method cannot give reliable information even on the energy scale that controls the low-temperature behaviour. This is on account of the two-stage Kondo effect that occurs in this case. As shown by Jayaprakash et al [13], the $S=1$ triplet is partially compensated by one of the parity channels and the residual spin is taken care of at a lower temperature, which itself represents the final scale characterizing the Fermi liquid ground state. However, the present method can predict the temperature scale on which the $S=1$ complex is partially compensated, namely

$$
T_{\mathrm{K}}^{\mathrm{eff}}=D(D / l)^{3\left[1+\cos \left(k_{\mathrm{F}} R\right) \mid / 2\left[1-\cos \left(k_{\mathrm{F}} R\right)\right]\right.} \exp \left[-1 / \rho J_{+}^{-}\right] .
$$

In deriving this expression we have taken the RKKY splitting to be much larger than the Kondo temperature. If we retain only the $\ln (\omega-I)$ terms and drop all the other logarithmic factors we obtain the exponential factor multiplied by the bandwidth. This exponential part is consistent with the results of Jayaprakash et al [13]. Keeping the remaining logarithms in (23) leads to the appearance of the prefactor in (24).

Turning now to the case of antiferromagnetic coupling we adopt the attitude that only one energy scale characterizes the Kondo compensation of each parity channel, and we study the dependence of the zeros of (23), representing as they do, the effective Kondo temperatures in each parity channel, as a function of the RKKY interaction $I$. In figures 2(a) and $(b)$ we plot the effective parity channel Kondo temperatures versus $I$ for various values of the parameter $\cos \left(k_{\mathrm{F}} R\right)$. If the cosine changes sign, even and odd parity are interchanged, so for convenience we shall restrict our attention to positive cosines. We find in all cases the expected asymmetry between even and odd-parity channels with a separation between $T_{\mathrm{K}}^{\text {eff }}$ 

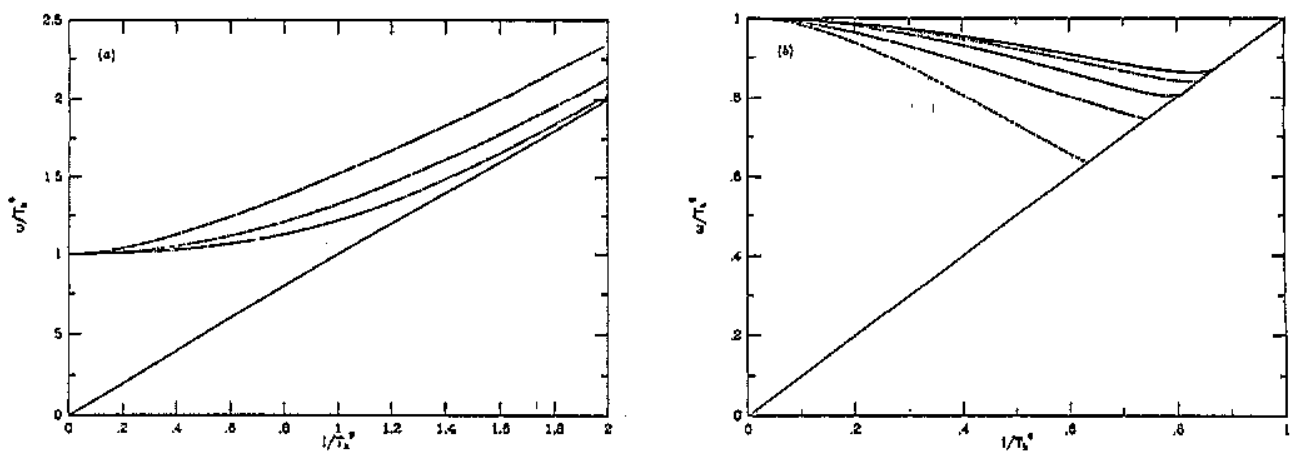

Figure 2. Variation of the effective Kondo temperature $\omega=T_{\mathrm{K}}^{\text {eff }}$ with RKKY splitting $/$ (both normalized to the Kondo temperature in the absence of the RKKY interaction, $\left.T_{K}^{D}\right)$ for $(a)$, evenparity and $(b)$ odd-parity states. In the former, $(a)$, the values of the parameter $\cos \left(k_{\mathrm{F}} R\right)$ are: for the dashed line, 0.1; for the short-dashed-dotted line, 0.3; and for the long-dashed-dotted line, 0.5. For the latcer, namely $(b)$ the odd-parity states, the values of $\cos \left(k_{F} R\right)$ are: for the full line, 0.5 ; for the short-dashed-dotted line, 0.3 ; for the long-dashed-dotted line, 0.7; for the long-dashed line, 0.1 ; and for the short-dashed line, 0.9 . In both figures the $\omega=l$ limit is also shown.

and $I$ decreasing as $I$ is increased. In one channel, for positive $\cos \left(k_{\mathrm{F}} R\right)$, the even-parity channel, this separation is always finite.

On the other hand we find that for the odd-parity channel the effective Kondo temperature crosses the $\omega=I$ line at a value $I=I_{\mathrm{cr}}$ of the order of the bare Kondo temperature $T_{\mathrm{K}}^{0}$. We can examine this crossing in more detail by extracting from (23) the coefficient multiplying the logarithm of $T_{\mathrm{K}}^{\mathrm{eff}}-I$. Equation (23) then becomes

$$
J_{+}^{-} a(I) \ln \left(\left(T_{\mathrm{K}}^{\mathrm{eff}}-I\right) / D\right)+b(I)=0
$$

where

$a(I)=1-\left(J_{+}^{-} / 2\right) \ln (I / D)+J_{+}^{-} \ln (2 I / D)-J_{+}^{+2} \ln ^{2}(I / D)-2 J_{+}^{-} J_{+}^{+} \ln ^{2}(2 I / D)$

and $b(I)$ is a more complicated but uninteresting expression. Solving (25) we obtain

$$
T_{\mathrm{K}}^{\mathrm{eff}}-I=D \exp \left[-b /\left(a J_{+}^{-}\right)\right]
$$

which has the form of a Kondo temperature apart from the factors $a$ and $b$. In the limit of large $I$ this yields the same expression as in the ferromagnetic case, and, were we dealing with a genuine crystal-field-split Kondo problem, the coefficients $a$ and $b$ would be constants, reflecting the fact that the exponent of the Kondo temperature depends on the residual coupling to the lowest crystal field multiplet.

However, in the present problem these are not constants and their dependence in $I$ reflects the fact that the residual coupling of conduction electrons to the lowest split state is affected by the interference of the coupling to higher-lying states. As a consequence of this interference the factor $a(I)$ vanishes at a critical value of $I$ given by

$$
I_{\mathrm{cr}}=D \exp \left[-1 /\left(2 \rho J f \cos \left(k_{\mathrm{F}} R\right)\right)\right]
$$

where $f\left(\cos \left(k_{\mathrm{F}} R\right)\right)$ is given by the solution of a simple quadratic equation and varies from a value for $\cos \left(k_{\mathrm{F}} R\right)=-1$ to a value for $\cos \left(k_{\mathrm{F}} R\right)$ equal to zero. The result of $a(I)$ 
vanishing is that the RHS of (27) becomes zero as soon as $I=I_{\mathrm{cr}}$. For negative $\cos \left(k_{\mathrm{F}} R\right)$ and even parity this root of (23) can be traced back onto the bare Kondo temperature as $I$ is reduced to zero. However, for positive $\cos \left(k_{F} R\right)$ the root given by (28), referring as it does, to the other parity channel, does not trace back onto $T_{\mathrm{K}}^{0}$ at $I=0$, explaining why only one parity channel has a vanishing effective Kondo temperature at $I=I_{\text {cr }}$.

\section{Conclusions}

The conclusion of this paper therefore is that for the two-impurity problem, with an antiferromagnetic interaction between the impurities, starting from zero RKKY and increasing the interaction the Kondo temperature of one of the electron parity channels decreases and ultimately vanishes when the RKKY interaction is of the order of the non-interacting Kondo temperature. Our calculation is restricted to particle-hole symmetry, i.e. we have not included potential scattering.

The consequences for the ground state are as follows: since the numerical studies find a phase shift of $\pi / 2$ in both of the parity channels $[11,14]$ we may conclude that the paritymixing part of the Hamiltonian will vanish at the unstable fixed point. The vanishing of one of the parity resonance widths found in this paper might then be reffected in a divergence of the low-temperature Fermi liquid parameters which are inversely proportional to the smallest energy scale involved. We should note, however, that our calculation retains only leading-order logarithms and that the higher-order next-leading logarithmic processes will affect some of the conclusions.

A number of open questions remain that require extensions of this work. Firstly, as regards potential scattering, it is straightforward to show that this leads to an extra term in the Hamiltonian that is different in the two parity channels. At leading logarithmic order it does not contribute, just as in the Kondo problem. However, this term does not commute with the parity-mixing term, and therefore will lead to effects at higher than leading logarithmic order.

Likewise the effects of renormalization of the RKKY interaction may be considered. We utilize the analogy with the crystal-field-split Kondo problem-since in the present representation the RKKY term is diagonal, in order to renormalize the RKKY interaction we have to examine self-energy corrections to the impurity Green functions that arise from the spin exchange terms in (1). For exactly the same reasons as in the ordinary Kondo problem, these involve one impurity propagator and two electron lines, and hence contribute nextleading logarithmic corrections to the RKKY interaction [16]. We are therefore justified in neglecting them in the present treatment.

The relevance of model (1) to real systems still remains an open question-namely the mention of only s-wave scattering, and the replacement of $k$ by the Fermi wavevector in the $k$-integrations. Nevertheless the calculations presented here give the first systematic high-energy treatment of (1), without being restricted to special coupling schemes $[5,6]$. While the retention of next-leading logarithmic terms is desirable, and probably essential near the scaling regime, the work describe here recovers the RKKY-induced destruction of the Kondo resonance-apparently a basic feature of the two-impurity Kondo problem.

\section{Acknowledgments}

This work was partially supported by US DOE grant DE-FG05-91ER45443. 


\section{References}

[1] Jones B A. Varma C M and Wikins J W 1988 Phys. Rev. Lett. 611415

[2] Ludwig A and Affleck I 1991 Phys. Rev. Lett. 61161

[3] Rasul J W and Schlottmann P 1989 Phys. Rev. Lett. 621701

[4] Nozieres P 1978 J. Physique Coll. 39 C6 1486

[5] Saso T 1990 Phys. Rev. B 44450

[6] Yanagisawa T 1991 J. Phys. Soc. Japan 6029

[7] Sakai O, Shimizu Y and Kasuya T 1990 Solid State Commun. 75 81; 1992 J. Phys. Soc. Japan 61 2333, 2348

[8] Fye R M and Hirsch J E 1989 Phys. Rev. B 404780

[9] Jones B A 199] Physica B 17153

[10] Jones B A, Kotliar B G and Millis A J 1989 Phys. Rev. B 393415

[11] Millis A J, Kotliar B G and Jones B A 1990 Field Theories in Condensed Matter Physics ed Z Tesanovich (Redwood City, CA: Addison Wesley) p 159

[12] Nagaoka X 1965 Phys. Rev. A 1381112

Sato K and Nagaoka Y 1973 Prog. Theor. Phys. 50 37; 1975 Prog. Theor. Phys. 53366

[13] Jayaprakash C, Krishna-Murthy H R and Wilkins J W 1981 Phys. Rev. Lett. 47737

[14] Jones B A and Varma C M 1989 Phys. Rev. B 40324

[15] Schlottmann P and Rasul J W 1992 Phys. Rev. B 454710

[16] Fowler M and Zawadowski A 1969 Solid State Commun. 9471 